\section{Insights into the SIRFLOX study}

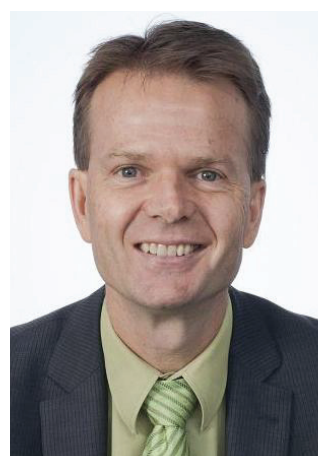

Peter Gibbs* speaks to Laura Dormer, Editorial Director: Peter Gibbs is a medical oncologist at the Western and Royal Melbourne Western Hospitals (Australia), and a laboratory head at the Walter and Eliza Hall Institute (Australia). He has a particular interest in translational research related to prognostic and predictive biomarkers for colorectal cancer. $\mathrm{He}$ is also heavily involved in clinical research, including being lead investigator of multiple international Phase III trials. He leads several national and international cancer registries, capturing comprehensive treatment and outcome data for patients treated in routine clinical care.

First draft submitted: 8 April 2016; Accepted for publication: 22 April 2016; Published online: 12 May 2016

\section{Q Could you briefly summarize your career path \& how you came to work in metastatic colorectal cancer?}

After completing my medical training in Melbourne (Australia), and spending 2 years in Denver, Colorado (USA), I was fortunate to return to work in Melbourne with a joint clinical and research role at the Ludwig Institute for Cancer Research and the Royal Melbourne Hospital. A focus of this role was colorectal cancer and my interest in this disease and involvement in a broad range of translational and clinical research initiatives developed from there.

\section{Q You are a clinician-scientist: how do you divide your time between clinical \& research work?}

It is challenging to find time to complete both roles, but also rewarding. A key to success is extensive collaboration, and most of my research involves working with scientific collaborators locally and internationally.

\section{Q What is the current standard of care for colorectal cancer hepatic metastases? What are the main unmet needs for metastatic colorectal cancer patients? \\ Unless patients are the minority with resectable disease, the standard approach is combi- nation chemotherapy and biologic, either bevacizumab or an EGF receptor inhibitor (if tumors are $K R A S$ wild-type). While this significantly improves patient outcomes overall there are some patients who do not benefit and, inevitably, with systemic therapy alone, the metastatic disease will become resistant to chemotherapy or patients will struggle to tolerate ongoing treatment.}

\section{Q As Co-Principal Investigator on the SIRFLOX study, could you briefly describe the main aims of this research?}

The main aim of the SIRFLOX study [1] was to see if the addition of selective internal radiation therapy (SIRT) to standard combination chemotherapy \pm bevacizumab could

*Western Hospital, Melbourne, Australia; peter.gibbs@mh.org.au

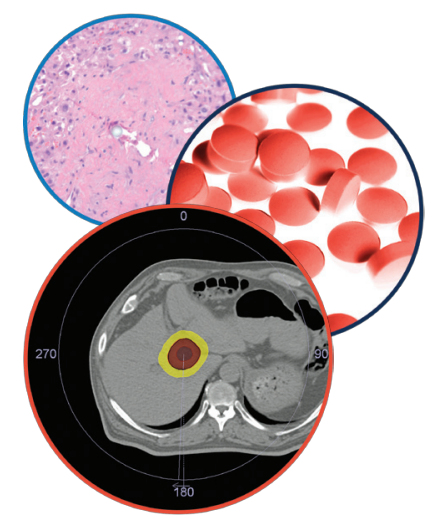

Hepatic Oncology

\section{KEYWORDS}

- colorectal cancer • liver metastases

- radioembolization • SIRT 
improve the duration of control of liver metastases, with the ultimate aim of improving overall survival, as for most of these patients the cause of death will be the liver metastases.

\section{Q Could you describe the patient population} studied?

These were previously untreated patients with metastatic colorectal cancer, where disease was confined to the liver or only a small amount of extrahepatic disease was present (with or without the primary tumor in situ). All patients had a good performance status and adequate major organ function.

\section{Q What were the key findings?}

That the addition of SIRT to standard systemic therapy significantly improved response rate in the liver and the duration of control of liver metastases, with no unexpected or unacceptable toxicity. There was no significant gain in overall progression, due to SIRT not impacting on extrahepatic disease.

\section{Q How did the safety profile compare with expectations?}

The observed toxicity was as expected. In particular, the rate of liver failure was low (not different to the control arm) and the rate of gastrointestinal ulceration was low, particularly later in the study as sites became more experienced.

Q The study showed that SIRT significantly extended progression-free survival in the liver by 7.9 months \& resulted in a 31\% reduction in risk of progression in the liver. What is the clinical significance of these findings?

This is still to be determined. There is an expectation that this gain in control of liver metastases will lead to a significant gain in overall survival, given that progressive liver metastases are the dominant cause of death in this patient population, but we are awaiting overall survival data.

Q What are the overall implications of the SIRFLOX study findings for the management of metastatic colorectal cancer?

This study has demonstrated that SIRT can safely be added to first-line chemotherapy, that this improves response rates in the liver and that this leads to prolonged control of liver metastases. Overall survival data are awaited, and this is required before SIRT becomes part of the standard first-line therapy for metastatic colorectal cancer.

Q What are the next steps for the research in this area? Where will your own research be focusing over the next few years?

We are currently awaiting the overall survival data from SIRFLOX and two other first-line studies. There are multiple opportunities to improve upon the results of the SIRFLOX study, but improved patient selection and a better understanding of the optimal timing of SIRT administration, and the optimal systemic therapy to combine with SIRT. Improvements in dosing and delivery of SIRT are also anticipated in the next few years.

\section{Q Do you have any final comments for the} readers of Hepatic Oncology?

It is important to remember the option of liverdirected therapies for patients with colorectal cancer liver metastases. Currently, SIRT has randomized trial data supporting use in the chemorefractory setting. Overall survival data for the first-line studies is anticipated in mid-2017.

\section{Disclaimer}

The opinions expressed in this interview are those of the interviewee and do not necessarily reflect the views of Future Medicine Ltd.

Financial \& competing interests disclosure The author has received honoraria from SIRTEX for attendance at advisory boards and for presentations. The author has no other relevant affiliations or financial involvement with any organization or entity with a financial interest in or financial conflict with the subject matter or materials discussed in the manuscript apart from those disclosed.

No writing assistance was utilized in the production of this manuscript.

\section{Reference}

1 van Hazel GA, Heinemann V, Sharma NK et al. SIRFLOX: randomized Phase III trial comparing first-line mFOLFOX6 (plus or minus bevacizumab) versus mFOLFOX6 (plus or minus bevacizumab) plus selective internal radiation therapy in patients with metastatic colorectal cancer. J. Clin. Oncol. 34(15), 1723-1731 (2016). 